\title{
Comparison of four off-the-shelf unmanned aerial vehicles (UAVs) and two photogrammetry programmes for monitoring pasture and cropping field trials
}

\author{
Michael R. Trolove* and Paul R. Shorten \\ AgResearch, Ruakura Research Centre, PB 3123, Hamilton 3240, New Zealand \\ ${ }^{*}$ Corresponding author: michael.trolove@agresearch.co.nz
}

\begin{abstract}
Rapid advancements in UAVs, computing power and photogrammetry techniques now permit low cost biological-monitoring applications using off-the-shelf hardware and software. The utility of four UAV models costing \$1,200 - \$11,000 and two photogrammetry programmes were assessed in separate experiments to evaluate their ability to detect standardised plant targets and to generate useable orthomoasic images. The colour and contrast of standardised targets influenced detection by UAVs more than their size as height increased. A large green rosette $\left(50.8 \mathrm{~cm}^{2}\right)$ could be detected by all UAV from $28-90$ $\mathrm{m}$, while a yellow target 13 times smaller could be detected at 36-100 m, with the more expensive UAVs being effective at the higher altitudes. Monitoring vegetation cover or flowering plants is possible at the minimum allowable height altitude of $20 \mathrm{~m}$ by all four UAVs. However, identification of species in their vegetative state would require the UAVs with the better camera optics. The two photogrammetry programmes produced suitable orthomosaic images under the pasture, maize and hill country scenarios tested.
\end{abstract}

Keywords Photogrammetry, orthomosaic, field trial assessment, vegetation cover

\section{INTRODUCTION}

The rapid advancement in recent years of unmanned aerial vehicles (UAVs) combined with photogrammetry techniques (Colomina \& Molina 2014) has opened up a wide variety of applications in the close-range aerial domain (Nex \& Remondino 2014). Applications where such imagery can be used are agriculture, forestry, archaeology, architecture, environmental monitoring, emergency management and traffic monitoring (e.g. Torres-Sánchez et al. 2013; Nex \& Remondino 2014; Whitehead et al. 2014; Panagiotidis 2019).

An advantage of UAVs is they can be deployed at low altitudes with low operator risk and operational costs compared to traditional aircraft platforms, while maintaining high accuracy (Nex \& Remondino 2014). When combined with photogrammetry software (that combines multiple images together into one image), UAVs and their light-weight cameras can produce a high-resolution orthomosaic image that is based on multiple images georeferenced to the earth's surface.

Flight altitude is critical in determining the final resolution of the orthomosaic image (Mesas-Carrascosa et al. 2015). This resolution needs to be sufficient to fulfil a specific objective. For example, fine-resolution images of general vegetation cover may not be required but identifying a species within a vegetation sward requires production of images with a finer spatial scale. Hengl (2006) established a general principle that at least four pixels are required to detect the smallest object within an image. Once a specific UAV and camera are selected the only variable to change is the survey altitude to achieve the specific resolution required for the 
object of interest.

Many flight-ready UAVs are now available that come complete with most components necessary for producing aerial orthomosaic images, notably: flight control via a remote control; a camera; built-in global positioning system (GPS) receiver(s) for georeferencing; and back-up safety features like Return To Home if batteries are low or radio transmission is lost. These set-ups are relatively small and low cost (usually $<\$ 10,000$ ) which makes them very accessible, but these have limited payload capacities. Larger units (generally $>\$ 10,000$ ) capable of larger payloads, also usually require the separate purchase of a sensor or sensors of the user's choice, which adds to the cost but can be purpose-built for specific objectives. For these reasons, three flight-ready UAV s costing less than $\$ 10,000$ and one costing $\$ 11,000$ were selected for evaluation.

This paper focuses on the feasibility of using flight-ready UAVs for monitoring and measurements of scientific agricultural field trials. Potentially, this technique has many added benefits for research work such as monitoring of: vegetation cover; plant populations and weed invasiveness; species identification (for example clover detection in a ryegrass sward); plant health indices; and many more. Such applications would serve to improve on the objectivity and repeatability of data acquisition usually obtained by visual assessment. These applications also provide the ability to expand the scope and capacity of field research.

The aim of this study was to compare four off- the-shelf UAVs alongside two photogrammetry systems to determine if the resulting aerial images would be suitable for obtaining empirical data at a suitable scale for some common objectives (e.g. vegetation cover and species detection) in pasture and cropping field research trials. These evaluations were simple and not intended to provide a complete comparison of all features of the UAVs, rather they are to provide an indication of usefulness of some flight-ready UAVs for the above aim and what value for money is obtained from the types evaluated. Also, the tests were aimed to evaluate principles of the photogrammetry system.

\section{MATERIALS AND METHODS}

Four flight-ready UAVs (Phantom 3 Pro, Phantom 4 Pro, Inspire 1 (X5R camera) and Inspire 2 (X5S camera)) were chosen for evaluation from a world leading UAV producer Dá-Jiāng Innovations Science and Technology Co. Ltd (DJI). These four UAVs provided a good cross section of DJI's flight-ready range and were easily accessible for evaluation at the time. The four types of UAV evaluated are shown in Figure 1 along with an approximate price $(\mathrm{NZ} \$)$ at the time of purchase, for comparison. The relevant specifications for each UAV and camera set-up are presented in Table 1. The Inspire 2 UAV had the best camera optics while the Phantom 4 UAV had the longest flight time of 30 minutes and the most obstacle avoidance sensors, which are aspects useful to the operator.

Two independent experiments were designed

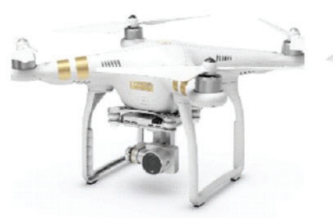

Phantom 3 Pro

$\$ 1,200$ (Jun 2015)

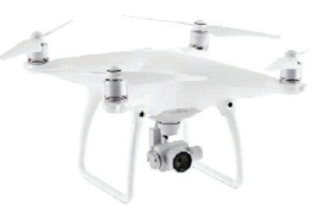

Phantom 4 Pro

$\$ 2,700(\operatorname{Jan} 2017)$

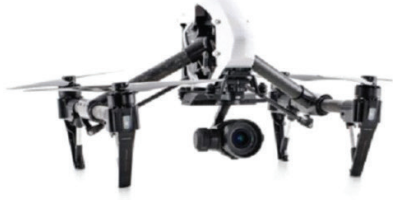

Inspire 1 Pro

(X5R camera)

$\$ 8,000(\operatorname{Sep} 2016)$

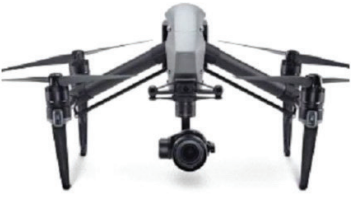

Inspire 2

(X5S camera)

$\$ 11,000$ (Feb 2018)

Figure 1 The four UAVs evaluated and an approximate price $(N Z \$)$ at the time of purchase for comparison. 
Table 1 Technical specifications for craft, battery and camera for each UAV.

\begin{tabular}{|c|c|c|c|c|}
\hline & Phantom 3 Pro & Phantom 4 Pro & Inspire 1 (X5R) & Inspire 2 (X5S) \\
\hline \multicolumn{5}{|l|}{ Aircraft } \\
\hline Flight time & approx. 23 minutes & approx. 30 minutes & approx. 18 minutes & approx. 25 minutes \\
\hline Vision system & Down & $\begin{array}{l}\text { Forward, back, } \\
\text { side \& down. }\end{array}$ & Down & Forward and down. \\
\hline Infrared system & None & $\begin{array}{l}\text { Horizontal \& } \\
\text { vertical }\end{array}$ & None & Upward \\
\hline \multicolumn{5}{|l|}{ Camera } \\
\hline $\begin{array}{l}\text { Sensor } \\
\text { dimensions (mm) }\end{array}$ & $\begin{array}{l}6.31748(\mathrm{~W}) \mathrm{x} \\
4.73811(\mathrm{H})\end{array}$ & $\begin{array}{l}12.8333(\mathrm{~W}) \mathrm{x} \\
7.21875(\mathrm{H})\end{array}$ & $\begin{array}{l}17.5(\mathrm{~W}) \mathrm{x} \\
13.125(\mathrm{H})\end{array}$ & $\begin{array}{l}17.5(\mathrm{~W}) \mathrm{x} \\
9.84375(\mathrm{H})\end{array}$ \\
\hline Pixel size $(\mu \mathrm{m})$ & 1.57937 & 2.34527 & 3.79774 & 3.31439 \\
\hline Effective pixels & $12.4 \mathrm{M}$ & $20 \mathrm{M}$ & $16 \mathrm{M}$ & $20.8 \mathrm{M}$ \\
\hline $\begin{array}{l}\text { Image size (pixels } \\
4: 3 \text { aspect ratio) }\end{array}$ & $4000 \times 3000$ & $4864 \times 3648$ & $4608 \times 3456$ & $5280 \times 3956$ \\
\hline Lens & $\begin{array}{l}\text { FOV } 94^{\circ} \\
20 \mathrm{~mm}(35 \mathrm{~mm} \text { eqv }\end{array}$ & $\begin{array}{l}\text { FOV } 84^{\circ} \\
24 \mathrm{~mm}(35 \mathrm{~mm} \text { eqv }\end{array}$ & $\begin{array}{l}\text { FOV } 72^{\circ} \\
15 \mathrm{~mm}(30 \mathrm{~mm} \text { eqv })\end{array}$ & $\begin{array}{l}\text { FOV } 72^{\circ} \\
15 \mathrm{~mm}(30 \mathrm{~mm} \text { eqv })\end{array}$ \\
\hline
\end{tabular}

and implemented. The first evaluated the four UAVs with their different camera specifications and the second compared two photogrammetry systems.

\section{Experiment 1: Evaluation of UAVs}

The four UAVs chosen were evaluated using a target-detection test. A series of 15 simulated plant targets representing the shapes of leaves and flowers of some common pasture species, i.e. clover (single leaflet and trifoliate leaf); buttercup; and a rosette (flat weed or thistle-like species). The targets were designed out of felt material as it had had low-reflectance properties. Various colours were used to evaluate how colour influenced detection and simulated leaves (green) and flowers (yellow, pink and purple). A total of six targets were yellow, four green and two each of purple and pink. The size of the targets was typical to the mimicked species and ranged from $0.7 \mathrm{~cm}^{2}$ for single clover leaflet target to $50 \mathrm{~cm}^{2}$ for the leaf rosette of a flat weed species (Fig. 2 ). These targets were then fastened on a brown hardboard sheet (Fig. 2). There was a single 0.7$\mathrm{mm}$ diameter red target on the board but data for this target are not shown. All four UAVs were flown over the target board at set intervals between $1-100 \mathrm{~m}$ above ground level (AGL), with images captured at each height interval, notably; 1 - $10 \mathrm{~m}$ (1 m intervals); 10 - $40 \mathrm{~m}$ (2 m intervals); $40-80 \mathrm{~m}$ (5 m intervals) and $80-100$ $\mathrm{m}$ (10 m intervals). All UAVs had functionality to adjust camera settings for exposure (EV) to account for different lighting conditions and exposure was set by each operator. The Inspire 2 UAV (the best in camera optics) was chosen and flown at two different exposures: a darker setting (Inspire 2D) where exposures were set relevant to the subject area - the target board - and a lighter exposure (Inspire 2L), accounting for the whole image (closer to auto-settings), to evaluate the influence of exposure on target detection. All UAVs were flown on the same day (3 May 2018). The day was fine with clear skies during all five flights, completed between 10:00 am - 1:30 pm. UAV flight height was calibrated with a metre ruler at the start (1-m interval) of each image capture run so variations in recorded height could be adjusted. Neither equipment nor flights were replicated for this simple, proof-of-concept test as this is likely to be how they are used in many practical applications.

The images obtained were assessed using image-analysis algorithms coded in MATLAB to ascertain how many simulated plant targets were visible at each height for each UAV. Reference 


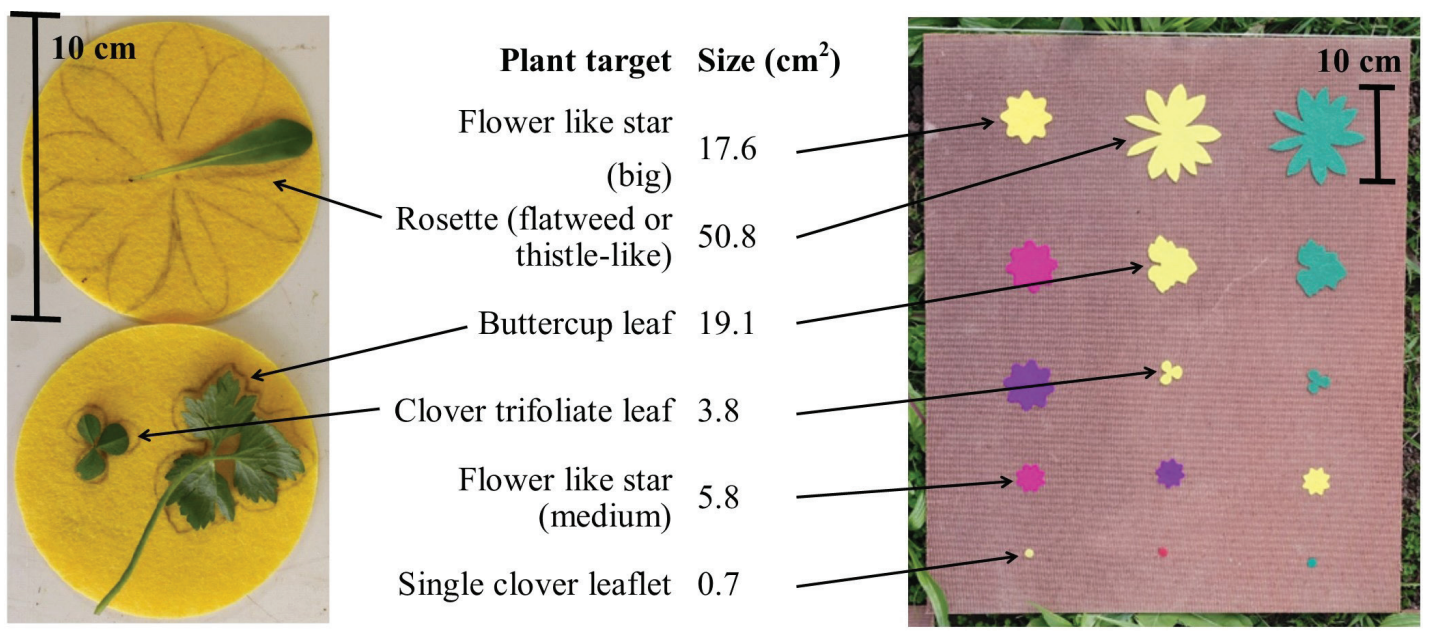

Figure 2 The simulated plant targets of various colours and their sizes. Design of the rosette, buttercup and clover trifoliate leaf (left) and plant targets as laid out on the $50 \mathrm{x} 60 \mathrm{~cm}$ hardboard sheet (right).

RGB colours for targets and the brown board were manually obtained from selected regions of the images at a target height of 2 metres because RGB is a device-dependent colour system. There was only a single red small sized target and for this reason it was excluded from analysis. The rectangular brown board region was manually selected for each image. The RGB spectra from each image were then classified based on the Euclidean distance of spectra to the reference spectra (Gonzalez et al. 2009). For each respective colour target, the connected components in the image were obtained (8 connected neighbourhood) (Gonzalez et al. 2009).

The area, filled area, perimeter, solidity, centroid and roundness were calculated for each connected component above a threshold size (Gonzalez et al. 2009). The threshold target size was $0.4 \mathrm{~cm}^{2}$ for yellow and green targets and $3.3 \mathrm{~cm}^{2}$ for pink and purple targets (due to the known minimum target size for each colour in the experiment). The area represented by 1 pixel was determined from the above-target height and properties of the UAV camera. Solidity is defined as the filled area divided by the convex area. Roundness is defined as $4 \pi$ multiplied by the filled area divided by the square of the perimeter. The filled area is defined as the region with holes filled. Visual observations of the occurrence of these target objects were also obtained from the captured UAV images.

\section{Experiment 2: Evaluation of image-processing software}

To produce an orthomoasic image, dedicated software beyond that supplied for normal UAV flight is required to determine the survey area, program the gridded flight path (waypoints) and camera trigger points needed to produce a series of overlapping images suitable for photogrammetry purposes. The DroneDeploy mapping platform (DroneDeploy, CA, USA) was selected from many available options as it satisfied the requirements of: flight plan; altitude; target area; and the route and waypoints necessary to complete the mission. Raw image data required further processing to obtain a single orthomosaic image. This process requires dedicated software and is fully automated based on fundamental principles of photogrammetry (Mesas-Carrascosa et al. 2015). Two suitable photogrammetry software programs (Pix4Dmapper (Lausanne, Switzerland) and Precision Mapper (PM; PrecisionHawk, USA)) were chosen as they satisfied the functionality required for these aerial surveys, ease of use and 
provided online support. Pix $4 \mathrm{D}$ is provided under a rental agreement or by out-right purchase, while PM can be utilised for free with a limited number of uses per month. These were evaluated under three 'typical use scenarios' of field trials. The scenarios undertaken are summarised below:

a. High-resolution, low-altitude flights at 3, 6 and $9 \mathrm{~m}$ AGL to detect a series of felt target objects laid over pasture.

b. Moderate altitude flights ( 15 - 30 m AGL) over a monoculture maize crop at three different stages of maturity and ground cover (7 leaves; 14 leaves; and mature plants at silage harvest time).

c. A high-altitude flight (50 m AGL) over a larger pastural area with hilly terrain (7.9 ha).

The Inspire 1 UAV was utilised to capture the image data sets for each scenario then each photogrammetry software was utilised to stitch these image sets together to form the georeferenced orthomosaic. The results were then evaluated based on accuracy of image features, such as detection of target objects, alignment of features (e.g. maize rows) and other distinct features within the survey area. Again, there was no replication in this preliminary study.

\section{RESULTS AND DISCUSSION}

\section{Experiment 1: UAV evaluation}

The target-detection test quantified the ability of the different UAVs to detect a range of targets that simulated pasture species. As expected, the number of detectible targets decreased in all images as the height of the UAV increased as shown in Figures 3A-E. This result was related to the size of the target within each colour type and image resolution. Smaller objects required a lower altitude and finer resolution to be detected than larger objects of the same colour. Green targets the size of a clover trifoliate leaf target could be distinguished up to $6 \mathrm{~m}$ (Phantom 3), 14 $\mathrm{m}$ (Phantom 4 and Inspire 2) and $22 \mathrm{~m}$ (Inspire 1). Larger green targets the size of buttercup, could be distinguished to $16 \mathrm{~m}$ by all crafts and up to $55 \mathrm{~m}$ by the Inspire 1 . The largest green rosette-shaped target could be distinguished to $28 \mathrm{~m}$ (Phantom 3) and $90 \mathrm{~m}$ (Inspire 1). A possible reason for the Inspire 1 performing the best in green target detection is its larger sensor size $(17.5 \times 13.125 \mathrm{~mm})$ compared to the other UAVs. This provides improved dynamic range and less diffraction, thereby improving clarity (Hamel 2019).

Yellow-coloured targets of equivalent size could be distinguished to greater altitudes than the green targets. The yellow clover trifoliate leaf target was detected at $\geq 36 \mathrm{~m}$ by all UAVs and up to $100 \mathrm{~m}$ (by the Inspire 2L). This height is approximately $4-7$ times higher than for the green trifoliate leaf target. Pink and purple coloured targets performed similarly with purple tending to be detected at higher altitudes in lighter exposed images (compare Inspire 2D with Inspire 2L, Figs. $3 \mathrm{D}$ and E). Overall, the Inspire 2 craft performed best across the yellow, pink and purple coloured targets, the Inspire 1, best for green targets. The UAV final ranking from most suitable to least was Inspire $2>$ Inspire $1>$ Phantom $4>$ Phantom 3 .

Target colour and its contrast in relation to its background was more important than size. The large green rosette $\left(50.8 \mathrm{~cm}^{2}\right)$ that had a low contrast relative to its background, could be distinguished by all UAVs from $28-90 \mathrm{~m}$, while the yellow clover trifoliate leaf target (3.8 $\mathrm{cm}^{2}$ ) some 13 times smaller and having a high contrast, could be detected to higher altitudes from $36-100 \mathrm{~m}$ by all UAVs. Hengl's (2006) size principle is a good starting point when planning an aerial survey, but colour and contrast of the target must also be considered before a final survey resolution is determined. Contrast was addressed by Hengl (2006) who stated that the greater the difference in reflectance values between the target to its surroundings the greater the detectability to higher altitudes. These results indicate that plant species with yellow flowers (the most common colour for flat weeds) could be detected at greater heights when flowering compared to their vegetative state. Note that, for this test, the prototype algorithm was designed to fulfil multiple size and colour classifications; 
A

Phantom 3

B Phantom 4

B Phantom 4
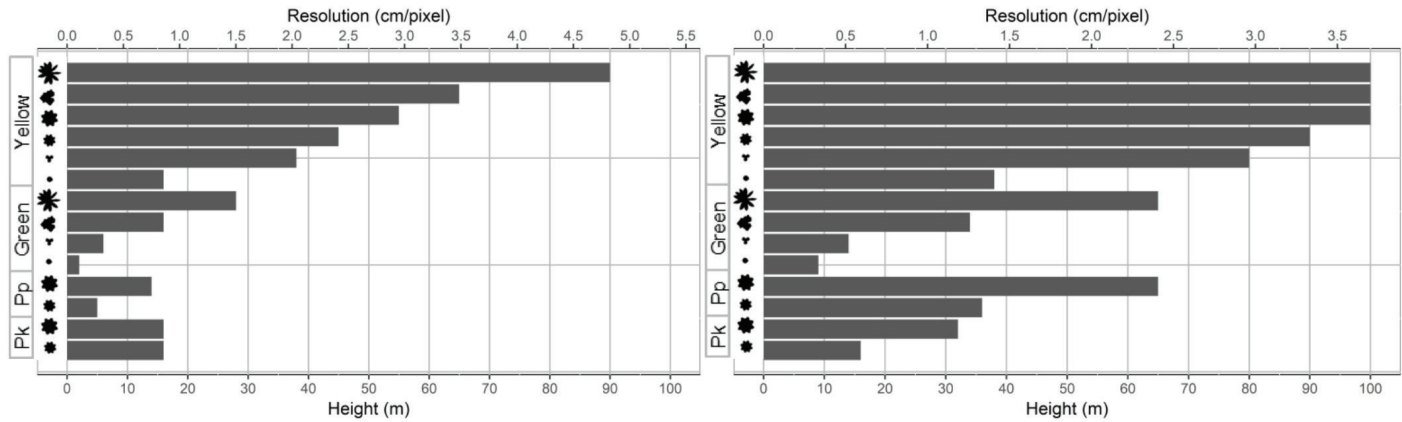

C

Inspire 1

D

Inspire 2D
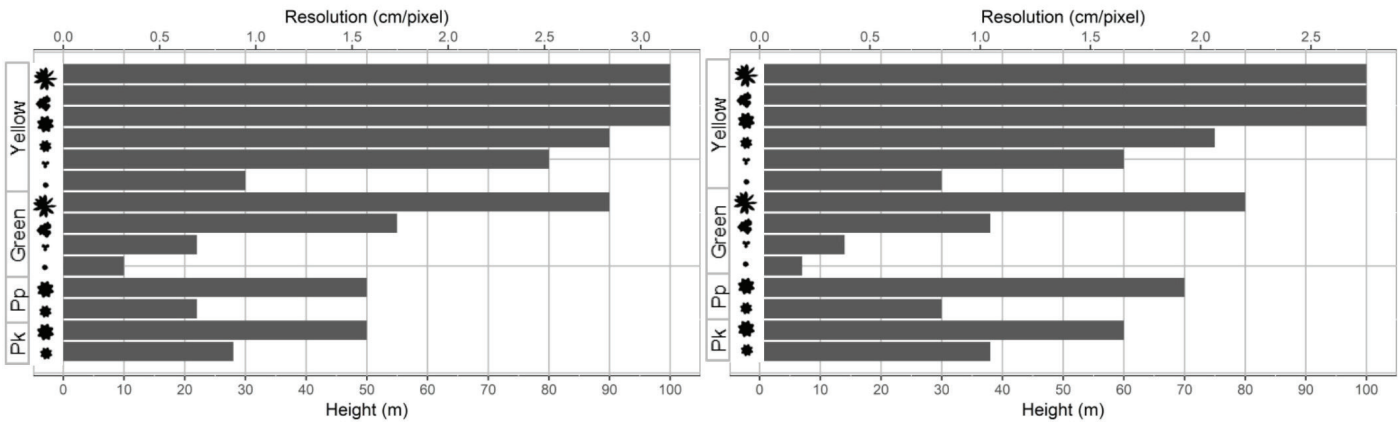

E

Inspire 2L

F
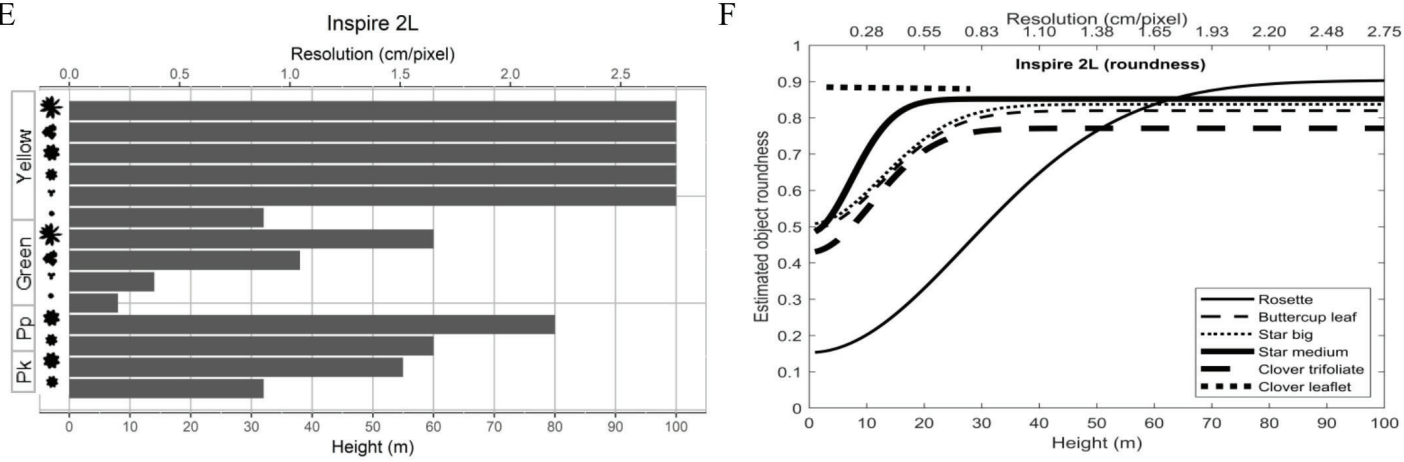

Figure 3 Maximum height at which each simulated plant target is detectible (out of 14) from 1-100 m, grouped according to colour for: Phantom 3 Pro (A); Phantom 4 Pro (B); Inspire 1 (C); Inspire 2 using a dark (D) exposure (D); and Inspire 2 using a light (L) exposure (E). Symbols represent the various plant target shapes as shown in Figure 2 and $\mathrm{Pp}=$ purple and $\mathrm{Pk}=$ pink. Object roundness (roundness $=1.0$ for a circular object) was estimated using data from Inspire $2 \mathrm{~L}$ for the six yellow targets of differing sizes at various heights $(\mathrm{F})$. 
however, an algorithm designed to fulfil a specific purpose (e.g. separation of the green clover trifoliate leaf) may exhibit a greater level of detectability. The detection heights based on visual observation and image processing were within 1-10 $\mathrm{m}$ of each other for all targets and heights, although visual observation tended to provide a 5-10 $\mathrm{m}$ detection advantage over the image processing-based estimate. This finding suggests that all UAVs apart from the Phantom 3 would be suitable to detect clover above the $20 \mathrm{~m}$ flight limit.

Image exposure also affected detectability depending on the contrast between subject and background. With high contrast (e.g. yellow target on brown background), a lighter exposure improved detection to higher altitudes such as for the Inspire 2D and 2L images where detection of the medium star and clover trifoliate leaf were increased from $75 \mathrm{~m}$ and $60 \mathrm{~m}$ to $100 \mathrm{~m}$ with the lighter exposure setting (Figs. 3D and 3E). However, the results were reversed in another test (data not presented). The darker exposed images enabled greater accuracy of detection of internal detail of targets to higher altitudes than the lighter exposed images, due to greater reflection from the lighter exposed images obscuring the internal detail. Thus, exposure, relative to the objective, also needs to be considered when setting image parameters.

Results of target shape in terms of solidity and roundness, correlated closely with image resolution. Targets retained their shape integrity to higher altitudes in images captured with the Inspire 1 and 2 crafts compared to the Phantom UAVs, even though cut-off points were at similar resolutions for all crafts. For example, in the Inspire $2 \mathrm{~L}$ images, the yellow rosette target retained a unique shape profile that was distinct from the yellow clover trifoliate leaf target until $50 \mathrm{~m}$ altitude (or $1.4 \mathrm{~cm}^{2} /$ pixel) when their lines converged (Fig. 3F). The other UAVs showed a similar cut-off point between these two targets at similar resolutions $\left(1.2-1.3 \mathrm{~cm}^{2} /\right.$ pixels $)$, yet to varying altitudes $(22-40 \mathrm{~m})$; data not shown. Therefore, if shape is a classifying feature used in image analysis to separate targets of like colours, then UAVs with better camera optics would increase detectability at higher altitudes. Here the rank from most to least suitable UAVs was Inspire $2>$ Inspire $1>$ Phantom $4>$ Phantom 3, which was also the order of decreasing price.

\section{Experiment 2: evaluation of image-processing software}

The results for Scenario (a) were obtained from low-level and high-resolution aerial surveys at 3,6 and $9 \mathrm{~m}$ respectively (Table 2 ). The survey at 3-m AGL took 35 minutes to complete (2 batteries/2 flights) and produced 759 images using an image overlap of $65 \% / 75 \%$ (side/front). However, neither type of image-processing software tested (Pix4D and PM) was able to produce an orthomosaic from this image set (Table 2). This failure was mainly due to the decrease in the field of view of each image at this low altitude and subsequent lack of distinct features across several images for tie points and image-stitching purposes. Both the Pix4D and PM systems were able to generate orthomosaics from data collected at either $6 \mathrm{~m}$ or $9 \mathrm{~m}$. Soon after many of these flight tests were carried out, a software update within the DroneDeploy software system (the flight controller program used) restricted flight altitudes to a minimum of $20 \mathrm{~m}$. The main reason for this change was craft safety (e.g. ability to clear most obstacles such as trees etc), plus reduced survey time (which increases dramatically below $20 \mathrm{~m}$ due to image-overlap requirements). Therefore, for final recommendations one needs to be mindful of this $20 \mathrm{~m}$ height threshold. Lower flying heights may be possible with a purpose-built setup or by contact with flight planning software developers.

The moderate-height flights over maize at different stages of maturity (Scenario b) produced different orthomosaic images depending on the maize growth stage and the area surveyed (Table 2). Both the Pix4D and PM systems produced an accurate orthomosaic from data collected at $15 \mathrm{~m}$ over 0.7 ha for maize at the 7-leaf growth stage. This result is likely due to the good contrast between the green plants and surrounding brown soil even though the plants were small. However, 
Table 2 Results of photogrammetry processing of aerial images taken over pasture or maize at various heights by Pix 4D and Precision Mapper (PM).

\begin{tabular}{|c|c|c|c|c|c|c|c|c|}
\hline \multirow[b]{2}{*}{$\begin{array}{c}\text { Height } \\
\operatorname{AGL}(\mathrm{m})\end{array}$} & \multirow[b]{2}{*}{$\begin{array}{l}\text { Flight } \\
\text { time } \\
(\mathrm{min})\end{array}$} & \multirow[b]{2}{*}{$\begin{array}{l}\text { Number } \\
\text { of } \\
\text { batteries } \\
\text { used } \\
\end{array}$} & \multirow[b]{2}{*}{$\begin{array}{l}\text { Number } \\
\text { of images } \\
\text { collected }\end{array}$} & \multirow[b]{2}{*}{ Area (ha) } & \multirow[b]{2}{*}{$\begin{array}{c}\text { Image } \\
\text { overlap } \\
\text { (side x } \\
\text { front; \%) }\end{array}$} & \multirow[b]{2}{*}{ Crop type } & \multicolumn{2}{|c|}{ Successful orthomosaic? } \\
\hline & & & & & & & Pix4D & $\mathrm{PM}$ \\
\hline 3 & 35 & 2 & 759 & 0.14 & $65 \times 75$ & $\begin{array}{l}\text { Felt objects } \\
\text { over pasture }\end{array}$ & No & No \\
\hline 6 & 17 & 1 & 252 & 0.14 & $65 \times 75$ & $\begin{array}{l}\text { Felt objects } \\
\text { over pasture }\end{array}$ & Yes & $\mathrm{No} / \mathrm{Yes}^{1}$ \\
\hline 9 & 7 & 1 & 114 & 0.14 & $65 \times 75$ & $\begin{array}{c}\text { Felt objects } \\
\text { over pasture }\end{array}$ & Yes & $\mathrm{No} / \mathrm{Yes}^{1}$ \\
\hline 15 & 9 & 1 & 191 & 0.7 & $60 \times 75$ & $\begin{array}{l}\text { Maize } \\
\text { (7 leaves. } \\
\text { Open } \\
\text { canopy) }\end{array}$ & Yes & Yes \\
\hline 15 & 15 & 1 & 462 & 0.7 & $75 \times 80$ & $\begin{array}{l}\text { Maize } \\
\text { (14 leaves. } \\
\text { Closed } \\
\text { canopy) }\end{array}$ & $\begin{array}{l}\text { Some areas } \\
\text { missing }\end{array}$ & $\begin{array}{l}\text { Some areas } \\
\text { missing \& } \\
\text { misaligned } \\
\text { maize rows }\end{array}$ \\
\hline 30 & 7 & 1 & 250 & 1.2 & $75 \times 80$ & $\begin{array}{l}\text { Maize } \\
\text { (Mature. } \\
\text { Closed } \\
\text { canopy) }\end{array}$ & $\begin{array}{l}\text { Yes. Some } \\
\text { leaf blur }\end{array}$ & $\begin{array}{c}\text { Yes. } \\
\text { Considerable } \\
\text { leaf blur }\end{array}$ \\
\hline 50 & 17 & 1 & 332 & 7.9 & $60 \times 75$ & $\begin{array}{l}\text { Pasture } \\
\text { (Hilly } \\
\text { terrain) }\end{array}$ & Yes & Yes \\
\hline
\end{tabular}

${ }^{1}$ No, when processed Oct 2017; Yes, when processed May 2018 (Due to an improvement in software by the provider).

the maize had a closed canopy by the 14-leaf stage, and neither image-processing system produced a completely error-free orthomosaic from data collected at $15 \mathrm{~m}$ over $0.7 \mathrm{ha}$, even though the recommended $\geq 75 \%$ overlaps for monocultures were used (DroneDeploy 2018). Images from both systems had missing areas around the edges, mainly due to those sections of the survey region having insufficient unique features for tie points (refer to Fig. 4A for a Pix4D example). In addition, the PM system had misaligned some maize rows. Homogeneity of cover and colour were expected for mature maize so the flight altitude was increased to $30 \mathrm{~m}$ and area surveyed was increased to 1.2 ha to ensure sufficient features around the field trial area were captured.
Both the Pix4D and PM systems produced an accurate orthomosaic from these data, although some leaf blur was visually evident, probably due to windy conditions and resulting leaf movement between successive images (refer to Fig. $4 \mathrm{~B}$ for a PM example). The PM system showed more leaf blur of the maize leaves than the Pix 4D system (data not shown).

Scenario (c) involved collecting data from a much larger area (7.9 ha) at a higher altitude (50 $\mathrm{m}$ ), over hilly terrain (Table 2). Both Pix 4D and PM systems produced accurate orthomosaics from the image data collected.

The results obtained here indicate that the following criteria are required to generate highquality orthomosaic images for monoculture 


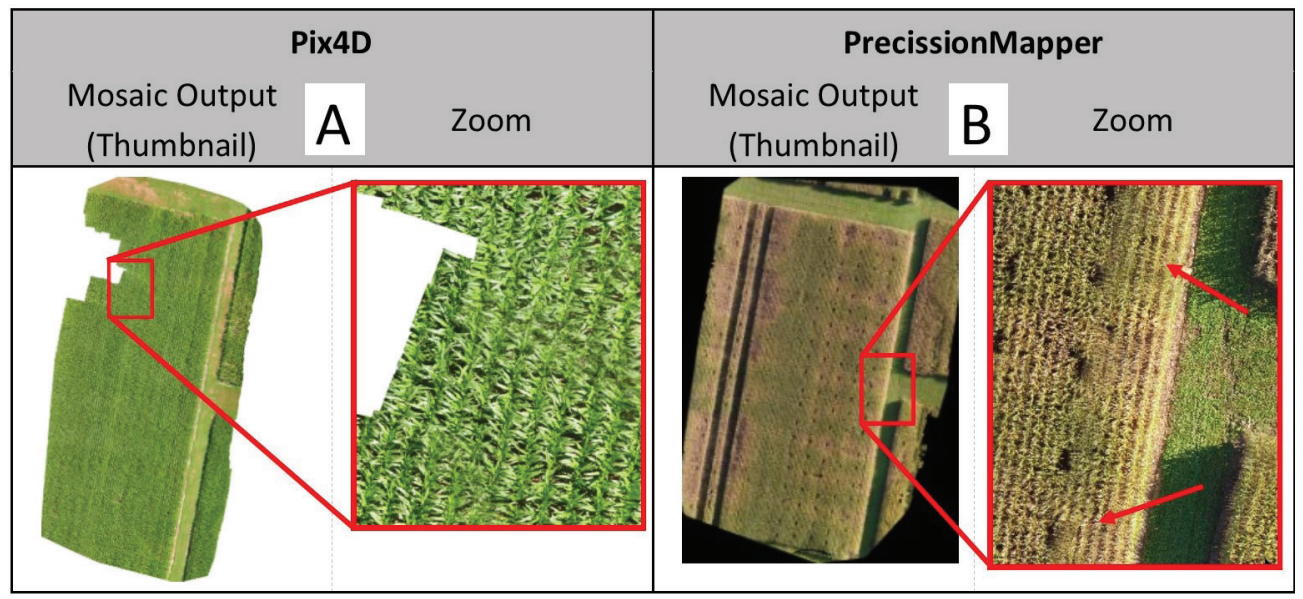

Figure 4 (A) A Pix4D orthomosaic produced from 462 images of maize at 14 leaves and a closed canopy, showing some incomplete sections; and (B) A complete PM orthomosaic produced from 250 images of mature maize and closed canopy, showing some areas of a lot of leaf blur as indicated by the arrows.

areas such as pasture or maize: set image overlap to $\geq 75 \%$ as recommended; and ensure that there are enough common features within/around the survey area to provide contrast and for sufficient tie points. These features can include hedges, fences, changes in vegetation, or bare soil around the survey area. The weather conditions or time of day that flights are undertaken also need to be considered in order to minimise image-production issues such as blur due to leaf movement.

\section{CONCLUSIONS}

In summary, all four UAVs evaluated would be suitable to use for applications such as determining vegetation cover on bare soil, identification of species when flowering (i.e. when contrast between background is greatest), or for distinguishing some larger rosette-type species $\left(>50 \mathrm{~cm}^{2}\right)$ in their vegetative (green) state while following the $20 \mathrm{~m}$ minimum flight limit of the flight controller program. However, a high number of camera pixels and sensor size are critical to improve image resolution and maintain image clarity when detecting smaller species like clover and separating species of similar colours. A minimum sensor size of $12.8 \mathrm{x}$
$7 \mathrm{~mm}$ (Phantom 4 Pro) would be recommended. To ensure successful orthomosaic images are obtained, it is recommended to increase the survey area beyond the target area to include distinct features, to use high overlaps ( $\geq 75 \%)$ over monocultures and to use auto-exposure with a compensation in EV if required. Both Pix4D and Precision Mapper proved suitable for orthomosaic processing.

\section{ACKNOWLEDGEMENTS}

We thank AgResearch and the Curiosity fund for the opportunity to undertake this project, Allister Holmes (Foundation for Arable Research) and Jaco Reyneke-Barnard (Ilota Limited) for flying their Phantom 4 UAVs as part of the evaluation tests, and Chris Buddenhagen (AgResearch) for reviewing the paper.

\section{REFERENCES}

Colomina I, Molina P 2014. Unmanned aerial systems for photogrammetry and remote sensing: A review. ISPRS Journal of Photogrammetry and Remote Sensing 92: 79-97.

DroneDeploy 2018. Supported Drones. https:// support.dronedeploy.com/docs/supported- 
drones (accessed 18 July 2018).

Gonzalez RC, Woods RE, Eddins SL 2009. Digital Image Processing using Matlab ( $2^{\text {nd }}$ Edition). Gatesmark Publishing. United States. 827 p.

Hamel J 2006 - 2019. 6 benefits of using cameras with larger sensors. Digital Photography School. from https://digital-photographyschool.com/6-benefits-of-using-cameraswith-larger-sensors/ (accessed 11 April 2019).

Hengl T 2006. Finding the right pixel size. Computers \& Geosciences 32: 1283-1298.

Nex F, Remondino F 2014. UAV for 3D mapping applications: a review. Applied Geomatics 6: $1-15$.

Mesas-Carrascosa FJ, Torres-Sánchez J, ClaveroRumbao I, García-Ferrer A, Peña JM, BorraSerrano I, López-Granados F 2015. Assessing optimal flight parameters for generating accurate multispectral orthomosaicks by UAV to support site-specific crop management. Remote Sensing 7: 12793-12814.

Panagiotidis D, Abdollahnejad A, Surový P and Kuželka K 2019. Detection of fallen logs from high-resolution UAV Images. New Zealand Journal of Forestry Science, 49, e2.

Torres-Sánchez J, López-Granados F, De Castro AI, Peña-Barragán JM 2013. Configuration and specifications of an unmanned aerial vehicle (UAV) for early site-specific weed management. PloS one 8, e58210.

Whitehead K, Hugenholtz CH, Myshak S, Brown O, LeClair A, Tamminga A, Barchyn, TE, Moorman B and Eaton B 2014. Remote sensing of the environment with small unmanned aircraft systems (UASs), part 2: Scientific and commercial applications. Journal of Unmanned Vehicle Systems, 2(3): 86-102. 